\title{
THE BORSUK-ULAM THEOREM AND BISECTION OF NECKLACES
}

\author{
NOGA ALON AND DOUGLAS B. WEST
}

\begin{abstract}
The Borsuk-Ulam theorem of topology is applied to a problem in discrete mathematics. A bisection of a necklace with $k$ colors of beads is a collection of intervals whose union captures half the beads of each color. Every necklace with $k$ colors has a bisection formed by at most $k$ cuts. Higherdimensional generalizations are considered.
\end{abstract}

1. Introduction. Suppose a necklace opened at the clasp has $2 n$ beads, chosen from $k$ colors and arranged in some order. Suppose there are $2 a_{i}$ beads of color $i$. A bisection of the necklace is a collection of nonoverlapping intervals of beads whose union captures half the beads of each color. The size of the bisection is the number of cuts that form the intervals of the bisection (the opening at the clasp is not counted). By passing gradually from a single large interval to its complement, it is easy to see that when there are two colors of beads, two cuts always suffice, regardless of how long the necklace is. It is natural to ask whether for every $k$ there is a number of cuts that similarly suffices, independent of $n$.

The problem of finding bisections of small size arises naturally when two mathematically oriented thieves steal a necklace with $2 a_{i}$ jewels of type $i$ and wish to divide it efficiently and fairly between them, wasting as little as possible of the metal in the links between the jewels. As remarked in [1], the solution of this problem also has some applications to VLSI circuit design.

Note that if the beads of each of the $k$ colors appear contiguously on the necklace, then the size of any bisection is at least $k$, since there must be a cut in each color. The main result of [4] is that every necklace with $k$ colors of beads has a bisection of size at most $k$. The proof given in [4] involves induction on $k$ for an analogous continuous problem, using detailed topological methods. Here we begin by giving a very short proof of this result using the Borsuk-Ulam theorem [2] (see also [3]). By rephrasing the problem in a way that allows the Borsuk-Ulam theorem to be applied, we avoid the necessity of detailed topological arguments. In addition, this method enables us to improve some of the results of $[\mathbf{4}]$ and to answer some of the questions raised there. In particular, we generalize the main result to higher dimensions.

In $\S 2$, we describe the continuous version of the problem and show that it generalizes the discrete one. In $\S 3$ we apply the Borsuk-Ulam theorem to solve the continuous problem and note the relation between our result and a theorem of Hobby

Received by the editors November 1, 1985.

1980 Mathematics Subject Classification (1985 Revision). Primary 68E99, 54H25, 55M20, 26 A99.

Research of the first author was supported in part by the Weizmann Fellowship for Scientific Research and by NSF grant DMS 8406100.

Research of the second author was supported in part by a Beckman Fellowship, by ONR Grant N00014-85K0570, and by NSF grant DMS 8504322 . 
and Rice [5] on $L_{1}$ approximation. During the completion of this manuscript, we learned that our approach is, in fact, very similar to that of Pinkus [7], who gave a short proof of the Hobby-Rice theorem. In $\S 4$ we discuss the problem of splitting the necklace into $m>2$ parts, and the problem of splitting the necklace in other proportions. This last problem is related to a well-known theorem of Liapounoff [6] in measure theory. In $\S 5$ we describe some generalizations to higher dimensions that reveal a somewhat surprising difference between odd and even dimensions.

2. The continuous problem. Let $I=[0,1]$ be the unit interval, and suppose that every point of $I$ has a color $i, 1 \leq i \leq k$, such that for each $i$ the set of points colored $i$ is measurable. Let us call such a coloring of $I$ an interval coloring. For convenience of expression, an interval coloring with $k$ colors is a $k$-coloring. Given an interval coloring, a bisection of size $r$ is a sequence of numbers $0=y_{0}<y_{1}<$ $\cdots<y_{r}<y_{r+1}=1$ such that $\bigcup\left\{\left[y_{i-1}, y_{i}\right]: i \equiv 0 \bmod 2\right\}$ captures precisely half the measure of each color. As in the discrete problem, size at least $k$ is required for any bisection of a $k$-coloring in which each color appears contiguously and colors occupy disjoint intervals. In the next section we apply the Borsuk-Ulam theorem to prove

PROPOSITION 2.1. Every interval $k$-coloring has a bisection of size at most $k$.

The statement of this continuous problem differs infinitesimally from that in [4]. Nevertheless, by the same argument as in [4], this proposition yields the bound of $k$ for the size of bisections of $k$-color discrete necklaces. Simply turn an opened necklace of $n$ beads into an interval coloring by partitioning $[0,1]$ into $2 n$ equal segments and coloring the $j$ th segment by the color of the $j$ th bead of the necklace. Proposition 2.1 guarantees a bisection using at most $k$ cuts, but the cuts need not occur at the endpoints of the $2 n$ segments. If there is a "bad cut" in the interior of a segment belonging to the $i$ th color, then there must be another such bad cut. We can slide one of the cuts to increase the measure captured for color $i$ and the other cut to decrease it by the same amount until one of the cuts reaches the boundary of its small segment. Therefore, induction on the number of bad cuts guarantees that we can correct the bad cuts to obtain a bisection that translates back into a bisection for the discrete problem.

As noted in [4], this also allows us to satisfy the jewel thieves when some colors appear an odd number of times. For whatever partition of the "extra" bead of each type that they decide is equitable, the appropriate rounding up or down of each $a_{i}$ can be achieved in the splitting up within the same bound of $k$ cuts.

3. Proof of Proposition 2.1. We need the following well-known result:

LEMMA 3.1 (BORSUK-Ulam [2]-SEE ALSO [3]). Let $f: S^{k} \rightarrow \mathbf{R}^{k}$ be a continuous function from the $k$-dimensional sphere $S^{k}\left(\right.$ in $\left.\mathbf{R}^{k+1}\right)$ to $\mathbf{R}^{k}$. Suppose that $f(\bar{x})=-f(-\bar{x})$ for all $\bar{x} \in S^{k}$. Then there exists $\bar{x} \in S^{k}$ such that $f(\bar{x})=0$.

Given an interval $k$-coloring of $[0,1]$, define a function $f: S^{k} \rightarrow \mathbf{R}^{k}$ as follows. Let $\bar{x}=\left(x_{1}, \ldots, x_{k+1}\right)$ be a point of $S^{k}$. Define $\langle z\rangle=z(\bar{x})=\left(z_{0}, \ldots, z_{k+1}\right)$ by $z_{0}=0, z_{j}=\sum_{i=1}^{j} x_{i}^{2}$ for $j \geq 1$. For $1 \leq j \leq k$, define $f_{j}(\bar{x})=\sum_{i=1}^{k+1} \operatorname{sign}\left(x_{i}\right) m_{j}(i)$, where $m_{j}(i)$ is the measure of the $j$ th color in the segment $\left[z_{i-1}, z_{i}\right]$. Finally, put 
$f(\bar{x})=\left(f_{1}(\bar{x}), \ldots, f_{k}(\bar{x})\right)$. Clearly, $f: S^{k} \rightarrow \mathbf{R}^{k}$ is a continuous function satisfying $f(\bar{x})=-f(-\bar{x})$ for all $\bar{x} \in S^{k}$. (It is amusing to note that in fact $f$ is constant on the positive orthant, yielding the full measure of each color.) By Lemma 3.1, there exists $\bar{x} \in S^{k}$ such that $f(\bar{x})=0$. Put $Z=\bigcup\left\{\left[z_{i-1}, z_{i}\right]: \operatorname{sign}\left(\bar{x}_{i}\right)=+1\right\}$. Since $f(\bar{x})=0, Z$ captures half the measure of each color. Since endpoints of the intervals in $Z$ occur only at the $z_{i}$, this bisection has at most $k$ cuts, which completes the proof of Proposition 2.1.

REMARK 3.2. This proof holds even if we replace the density functions of the colors by arbitrary continuously-integrable functions. We thus get the following result, which improves Theorem 6 of [4] and was proved by Hobby and Rice in [5] (see also [7]).

Proposition 3.3 [5]. Let $g_{1}, \ldots, g_{k}:[0,1] \rightarrow \mathbf{R}$ be $k$ continuously-integrable functions. Then there exist $0=z_{0} \leq z_{1} \leq \cdots \leq z_{k} \leq z_{k+1}=1$ and $\delta_{1}, \ldots, \delta_{k+1} \in$ $\{ \pm 1\}$ such that $\sum_{i=1}^{k+1} \delta_{i} \int_{z_{i-1}}^{z_{i}} g_{j}=0$ for all $1 \leq j \leq k$.

4. Splitting in other proportions. Given an interval $k$-coloring, $k$ cuts suffice to form two disjoint sets each containing exactly half of each color. A natural question, raised in [4], is to consider $m$ thieves rather than two. In other words, determine for $m>2$ the minimum number of cuts $c(m, k)$ that always suffice to form $m$ disjoint sets each capturing $1 / m$ of each color. If every color appears contiguously, we must have at least $m-1$ cuts in each color, so $c(m, k) \geq(m-1) k$. Repeated application of Proposition 2.1 shows that when $m$ is a power of 2 this bound is best possible.

THEOREM 4.1. $c\left(2^{j}, k\right)=\left(2^{j}-1\right) k$.

PROOF. It suffices to show $c\left(2^{j}, k\right) \leq\left(2^{j}-1\right) k$, which we do by induction on $j$. For $j=1$ this is Proposition 2.1. For $j>1$, we apply the induction hypothesis for $j-1$ and 1 . Begin by using $\left(2^{j-1}-1\right) k$ cuts to form $2^{j-1}$ sets of intervals each containing $1 / 2^{j-1}$ of each color. For each of those sets, consider the interval coloring formed by placing those intervals next to each other and rescaling to total length 1 . Using at most $k$ cuts, this interval coloring can be bisected. Translating back to the original interval coloring, this adds altogether at most $k 2^{j-1}$ more cuts, so that the desired distribution is attained with at most $\left(2^{j}-1\right) k$ cuts.

This suggests the following

CONJECTURE 4.2. For every $m$ and $k, c(m, k)=(m-1) k$.

This conjecture is plausible because, besides holding for all $k$ when $m$ is a power of 2 , it also holds for all $m$ when $k=2$. In fact, for $k=2$ the following stronger result holds, yielding Conjecture 4.2 for $k=2$ by applying it succesively with $\alpha=1 / m, 1 /(m-1), \ldots, \frac{1}{2}$ and breaking off the amount due each thief one by one.

PROPOSITION 4.3. For every interval coloring with two colors and every $0<$ $\alpha<1$, one can form with at most two cuts a set of intervals that captures the fraction $\alpha$ from each of the two colors.

ProOF. View the points of the interval as real numbers modulo 1 (i.e., think of it as a cycle formed by identifying the endpoints). For $0 \leq x<1$, let $g(x)$ be 
the measure of color 1 in the interval $[x, x+\alpha(\bmod 1)]$. If $t$ is the total measure of color 1 in the interval, then the average value of $g(x)$ is $\int_{0}^{1} g(x) d x=\alpha t$. Since $g$ is continuous, $g(x)=\alpha t$ for some $\hat{x}$. Therefore, cutting at $\hat{x}$ and at $\hat{x}+\alpha(\bmod 1)$ yields a set of measure $\alpha$ containing the fraction $\alpha$ of the measure of color 1 ; hence it also contains $\alpha$ of the measure of color 2 .

By analogy with $c(m, k)$, let $c(\alpha, k)$ be the minimum number of cuts that suffices, in any interval $k$-coloring, to obtain a collection of intervals capturing $\alpha$ of the measure of each color. We do not know whether $c(\alpha, k)$ is well defined, i.e. finite, for every $k \geq 3$ and $0<\alpha<1$. In particular, we do not know this for $k=3$ and $\alpha=\frac{1}{3}$. By Theorem 4.1, $c(\alpha, k)$ exists whenever $\alpha=i / 2^{j}$, and Conjecture 4.2 would imply that it does at least when $\alpha$ is rational. When $A$ is not restricted to be the union of finitely many intervals, it is true that there always exists some measurable subset $A$ of the unit interval that captures $\alpha$ of the measure of each color. Indeed, this remains true when the colors are replaced by arbitrary continuous measures. This follows immediately from the following well-known result of Liapounoff [6].

LEMMA 4.4. Let $\mu_{1}, \ldots, \mu_{k}$ be $k$ continuous probability measures on $[0,1]$. For a measurable set $X \subseteq[0,1]$, let $\mu_{i}(X)$ be the measure of $\mu_{i}$ in $X$, and put $f(X)=$ $\left(\mu_{1}(X), \ldots, \mu_{K}(X)\right) \in \mathbf{R}^{k}$. Then $\{f(X): X \subseteq[0,1], X$ measurable $\}$ is a closed convex subset of $\mathbf{R}^{k}$.

In particular, since $(0, \ldots, 0)=f(\varnothing)$ and $(1, \ldots, 1)=f([0,1])$, there is some measurable $X$ whose image is $(\alpha, \ldots, \alpha)$.

5. Higher dimensions. Now we return to the case of 2 thieves, but consider higher-dimensional versions of the necklace. Let $C=C^{d}$ be the $d$-dimensional unit cube, and suppose the points of $C$ are colored using $k$ colors, such that the set of points with color $i$ is measurable for each $i$. Call this a $k$-coloring of the cube. Our results thus far easily imply that, given any direction, there are always at most $k$ hyperplanes perpendicular to that direction that form two disjoint sets of slices of $C$ each containing half of each color. For example, for the direction of the $x_{1}$-axis, this follows immediately by applying Proposition 3.3 (the Hobby-Rice theorem) to the functions $\left\{g_{j}: 1 \leq j \leq k\right\}$, where $g_{j}(y)$ is the $(d-1)$-dimensional measure of the $j$ th color in $C \cap\left\{\bar{x}: x_{1}=y\right\}$. Thus it is more interesting to consider bisections where we cut all the axes in the same way and 2-color the regions so formed like a $d$ dimensional checkerboard. In particular, given a sequence of numbers $\langle z\rangle$ such that $0=z_{0}<z_{1}<\cdots<z_{r}<z_{r+1}=1$, let $I_{i}=\left[z_{i-1}, z_{i}\right]$ and $C_{i_{1}, \ldots, i_{d}}=I_{i_{1}} \times \cdots \times I_{i_{d}}$. Then a bisection having size $r$ for a colored $d$-dimensional cube $C$ is a sequence $\langle z\rangle$ such that the set $\bigcup\left\{C_{i_{1}, \ldots, i_{d}}: i_{1}+\cdots+i_{d} \equiv 0(\bmod 2)\right\}$ captures precisely half the measure of each color.

Note that for $d=1$ this reduces to our previous definition of bisection. For $d=2$, a bisection corresponds to a black-white checkerboard with the same number of rows and columns, such that all the rectangles along the diagonal are squares and the white rectangles together contain half the measure of each color.

Somewhat surprisingly, it turns out that for odd dimensions there is always a bisection whose size does not exceed the number of colors, but for even dimensions, there is no finite size that always suffices to yield a bisection, even with only two colors. The remainder of the paper is devoted to proving these two results. 
THEOREM 5.1. If $d$ is odd, then every $k$-coloring of $C^{d}$ has a bisection of size at most $k$, and this bound is best possible.

ProOF. First we show that the bound, if true, is best possible. Consider any coloring of $C^{d}$ in which each of the first $k-1$ colors fills a small cube such that no hyperplane parallel to an axis cuts two of these cubes and all of them are close to the origin. The $k$ th color fills the rest of the cube. For example, for some small $\varepsilon>0$, and $1 \leq i \leq k$, let the $i$ th color fill the cube $[(i-1) \varepsilon, i \varepsilon] \times \cdots \times[(i-1) \varepsilon, i \varepsilon]$. Any bisection must contain some $z_{j}$ in the range $((i-1) \varepsilon, i \varepsilon)$, or else the $i$ th color is not bisected. Also, if $\varepsilon$ is sufficiently small, any bisection must contain some $z_{j}$ bigger than $k \varepsilon$, since otherwise a portion of the $k$ th color with measure $(i-(k-1) \varepsilon)^{d}>\frac{1}{2}$ has no cuts in it. Thus any bisection has size at least $k$.

The proof that any coloring has a bisection of size at most $k$ is similar to our proof of Proposition 2.1. Given $d$ odd and a $k$-coloring of $C^{d}$, define a function $f: S^{k} \rightarrow \mathbf{R}^{k}$ as follows. Let $\bar{x}=\left(x_{1}, \ldots, x_{k+1}\right)$ be a point of $S^{k}$. As in $\S 3$, define $z=z(\bar{x})=\left(z_{0}, \ldots, z_{k+1}\right)$ by $z_{0}=0, z_{j}=\sum_{i=1}^{j} x_{i}^{2}$ for $j \geq 1$. With $C_{i_{1}, \ldots, i_{d}}$ defined from $z$ as above, put

$$
f_{j}(\bar{x})=\sum_{i_{1}} \cdots \sum_{i_{d}} \operatorname{sign}\left(x_{i_{1}}\right) \cdots \operatorname{sign}\left(x_{i_{d}}\right) m_{j}\left(C_{i_{1} \ldots, i_{d}}\right),
$$

where $m_{j}\left(C_{i_{1}, \ldots, i_{d}}\right)$ is the measure of the $j$ th color in $C_{i_{1}, \ldots, i_{d}}$. Finally, let $f(\bar{x})=$ $\left(f_{1}(\bar{x}), \ldots, f_{k}(\bar{x})\right)$. Clearly, $f: S^{k} \rightarrow \mathbf{R}^{k}$ is a continuous function, and $f(\bar{x})=$ $-f(-\bar{x})$ for all $\bar{x} \in S^{k}$ since $d$ is odd. By Lemma 3.1 (the Borsuk-Ulam theorem), there exists $\bar{x} \in S^{k}$ such that $f(\bar{x})=0$. As in $\S 3$, this implies that $\langle z\rangle$ is a bisection.

Finally, we show that there is no analogue of Theorem 5.1 when the number of dimensions is even.

THEOREM 5.2. For every even $d \geq 2$ and every positive integer $l$, there is a 2-coloring of $C^{d}$ that has no bisection of size at most $l$.

PROOF. The main idea of the construction is that in any bisection the main diagonal $\left(\left\{\bar{x}: x_{1}=\cdots=x_{d}\right\}\right)$ lies in $\bigcup\left\{C_{i_{1}, \ldots, i_{d}}: i_{1}=\cdots=i_{d}\right\}$, and since $d$ is even these all satisfy $\sum i_{j} \equiv 0(\bmod 2)$ and are captured by the same thief. If the coloring is designed so that a color is concentrated near the main diagonal, these cubes will contain more than half its total measure.

For simplicity, we provide the details of the argument only for $d=2$; the general case is analogous. Given a positive integer $l$, choose $\varepsilon>0$ sufficiently small such that

$$
2 \varepsilon-(3 l+1) \varepsilon^{2}>\frac{1}{2}\left(2 \varepsilon_{-}-\varepsilon^{2}\right) .
$$

Color $C^{2}$ red and blue by letting $\left\{(x, y) \in C^{2}: x-\varepsilon \leq y \leq x+\varepsilon\right\}$ be red and all other points be blue.

Suppose $\langle z\rangle$ is a bisection of size $m$. Let $\delta_{i}=z_{i}-z_{i-1}$ for $1 \leq i \leq m+1$, and let $C_{i, j}$ be the regions generated by $\langle z\rangle$. If $\delta_{i} \geq \varepsilon$, then $C_{i, i}$ contains precisely $2 \varepsilon \delta_{i}-\varepsilon^{2}$ red area. Hence the total red area in $\bigcup C_{i, i}$ is at least $\sum_{\left\{i: \delta_{i} \geq \varepsilon\right\}}\left(2 \varepsilon \delta_{i}-\varepsilon^{2}\right)$. However, $\sum_{i=1}^{m} \delta_{i}=1$, and thus $\sum_{\left\{i: \delta_{i} \geq \varepsilon\right\}} \delta_{i}>1-m \varepsilon$. This implies that the red area in $\bigcup C_{i, i}$ exceeds $2 \varepsilon(1-m \varepsilon)-(m+1) \varepsilon^{2}=2 \varepsilon-(3 m+1) \varepsilon^{2}$. The total red area is $2 \varepsilon-\varepsilon^{2}$. By the definition of $\varepsilon$, if $m \leq l$, then $\bigcup C_{i, i}$ contains more than half of this. Hence there is no bisection of size at most $l$. 
NOTE ADDED IN PROOF. The first author has recently proved Conjecture 4.2. This will appear in [8].

\section{REFERENCES}

1. S. N. Bhatt and C. E. Leiserson, How to assemble tree machines, Proc. 14th ACM Sympos. Theor. Computing, San Francisco, Assoc. Comp. Mach., 1981, pp. 99-104.

2. K. Borsuk, Drei Satze uber die n-dimensionale eudlidische Sphare, Fund, Math. 20 (1933), 177190.

3. J. Dugundji, Topology, Allyn and Bacon, Boston, Mass., 1966, pp. 347-349.

4. C. H Goldberg and D. B. West, Bisection of circle colorings, SIAM J. Algebraic Discrete Methods 6 (1985), 93-106.

5. C. R. Hobby and J. R. Rice, A moment problem in $L_{1}$ approximation, Proc. Amer. Math. Soc. 16 (1965), 665-670.

6. A. Liapounoff, Sur les fonctions vecteurs completement additives, Izv. Akad. Nauk SSSR 4 (1940), 465-478.

7. A. Pinkus, A simple proof of the Hobby-Rice theorem, Proc. Amer. Math. Soc. 60 (1976), 82-84.

8. N. Alon, Splitting necklaces, Adv. in Math. (to appear).

Department of Mathematics, Massachusetts institute of Technology, CAMBRIDGE, MASSACHUSETTS 02139

Department of Mathematics, Tel-Aviv University, Tel-Aviv, Israel (Current address of Noga Alon)

DEPARTMENT OF MATHEMATICS, UNIVERSity OF ILlinOIS, URBANA, ILLINOIS 61801 (Current address of D. B. West) 